\title{
Active reset of a radiative cascade for entangled-photon generation beyond the continuous-driving limit
}

\author{
Jonathan R. A. Müller $\odot,{ }^{1,2}$ R. Mark Stevenson, ${ }^{1,}$ Joanna Skiba-Szymanska, ${ }^{1}$ Ginny Shooter $\odot,{ }^{1,3}$ Jan Huwer,${ }^{1}$ Ian Farrer $\odot,{ }^{3, \dagger}$ \\ David A. Ritchie, ${ }^{3}$ and Andrew J. Shields ${ }^{1}$ \\ ${ }^{1}$ Toshiba Research Europe Limited, Cambridge Research Laboratory, 208 Science Park, Milton Road, Cambridge CB4 OGZ, United Kingdom \\ ${ }^{2}$ Department of Physics and Astronomy, University of Sheffield, Hounsfield Road, Sheffield S3 7RH, United Kingdom \\ ${ }^{3}$ Cavendish Laboratory, University of Cambridge, Madingley Road, Cambridge CB3 OHE, United Kingdom
}

(Received 24 January 2020; revised 16 October 2020; accepted 22 October 2020; published 30 November 2020)

\begin{abstract}
The emission rate of entangled photons from cascaded few-level systems is limited by the dynamics of the radiative transitions. Here, we overcome the continuous driving limit for a semiconductor quantum dot via an active reset of the radiative cascade. We show theoretically and experimentally the driving regime to enable the generation of entangled photon pairs with higher fidelity and emission rate than the optimum continuously driven state. Finally, we electrically generate entangled photon pairs with an average fidelity of $(79.5 \pm 1.1) \%$ at a record clock rate of $1.15 \mathrm{GHz}$.
\end{abstract}

DOI: 10.1103/PhysRevResearch.2.043292

\section{INTRODUCTION}

Single and entangled photons promise unique advantages for many applications in quantum photonics, including enhanced secure key rates in quantum key distribution $(\mathrm{QKD})$ through elimination of multiphoton emission [1], and global scale unconditionally secure networks with entanglementbased quantum repeaters [2]. Semiconductor quantum dots (QDs) have been shown to emit single and entangled photons with approaching ideal properties [3-8], with the prospect of on-chip integration $[9,10]$, and compatibility with optical fibre quantum networks $[11,12]$. However, state-of-the-art QKD systems based on coherent laser pulses employ clock rates in the gigahertz range for high data throughput $[13,14]$. Clock rates achieved with entangled photons from QDs are much lower $[15,16]$, impeding high frequency operation. In this paper, we show that by pumping the system only for a fraction of the time, it is possible to access a regime where more entangled photon pairs are generated compared to keeping the system in an optimum continuously driven equilibrium state. This is made possible by employing a high-frequency pulsed excitation regime where the cycle is reset while still highly optically active, despite a reduced emission probability per clock cycle. At the same time, this scheme maintains a superior entanglement fidelity. We expect our results will enable improved performance of a wide range of entanglement-based photonic applications.

\footnotetext{
*mark.stevenson@crl.toshiba.co.uk

†resent address: Department of Electronic and Electrical Engineering, University of Sheffield, Mappin Street, Sheffield S1 3JD, United Kingdom.

Published by the American Physical Society under the terms of the Creative Commons Attribution 4.0 International license. Further distribution of this work must maintain attribution to the author(s) and the published article's title, journal citation, and DOI.
}

\section{THEORETICAL FRAMEWORK}

We begin by considering the excitation and radiative cascade dynamics of an atomic three-level system [17], as in this case, of a QD. The biexciton state $(X X)$ decays via an intermediate neutral exciton $(X)$ superposition state, resulting in two consecutively emitted, polarization-entangled photons [Fig. 1(a)] $[18,19]$. We explore this system using a rate equation model (see Appendix A), with the two consecutive transitions assigned radiative lifetimes of $\tau_{X X}=300 \mathrm{ps}$ and $\tau_{X}=500 \mathrm{ps}$, respectively, similar to observed experimentally. Figure 1(b) shows the modeled biexciton, exciton, and ground state $(\mathrm{G})$ population, driven at a slow $250 \mathrm{MHz}$ clock rate via $50 \mathrm{ps}$ rectangular electrical initialization pulses. When the QD is excited to the $X X$ state in the limit of low driving frequency, practically complete radiative decay of the QD emission is observed, generating an entangled photon pair each clock cycle. However, a substantial fraction of the clock cycle is spent at the tail of the cascade with high ground state population and low emission brightness.

In contrast, for continuous driving [Fig. 1(c)] the QD is in equilibrium, with the $X X$ and $X$ states always populated and an always nonzero emission intensity. However, at the DC power that maximizes the entangled pair emission rate (here referred to as "optimal DC") the distribution of the QD population in the DC equilibrium means that only a minority (43\%) of the emitted photons form part of a biexciton-exciton cascade, resulting in a reduced relative fraction of entangled pair emission.

We introduce a novel active reset (AR) driving scheme based on two core considerations: Firstly, to actively reset entanglement at a given clock cycle, the QD does not need to return to the ground state. Instead, it is sufficient to reinitialize the QD directly to the biexciton state- the initial state of the biexciton-exciton cascade. Secondly, resetting the QD before the population has fully cascaded to the ground state allows for an increased entangled-pair emission brightness, 

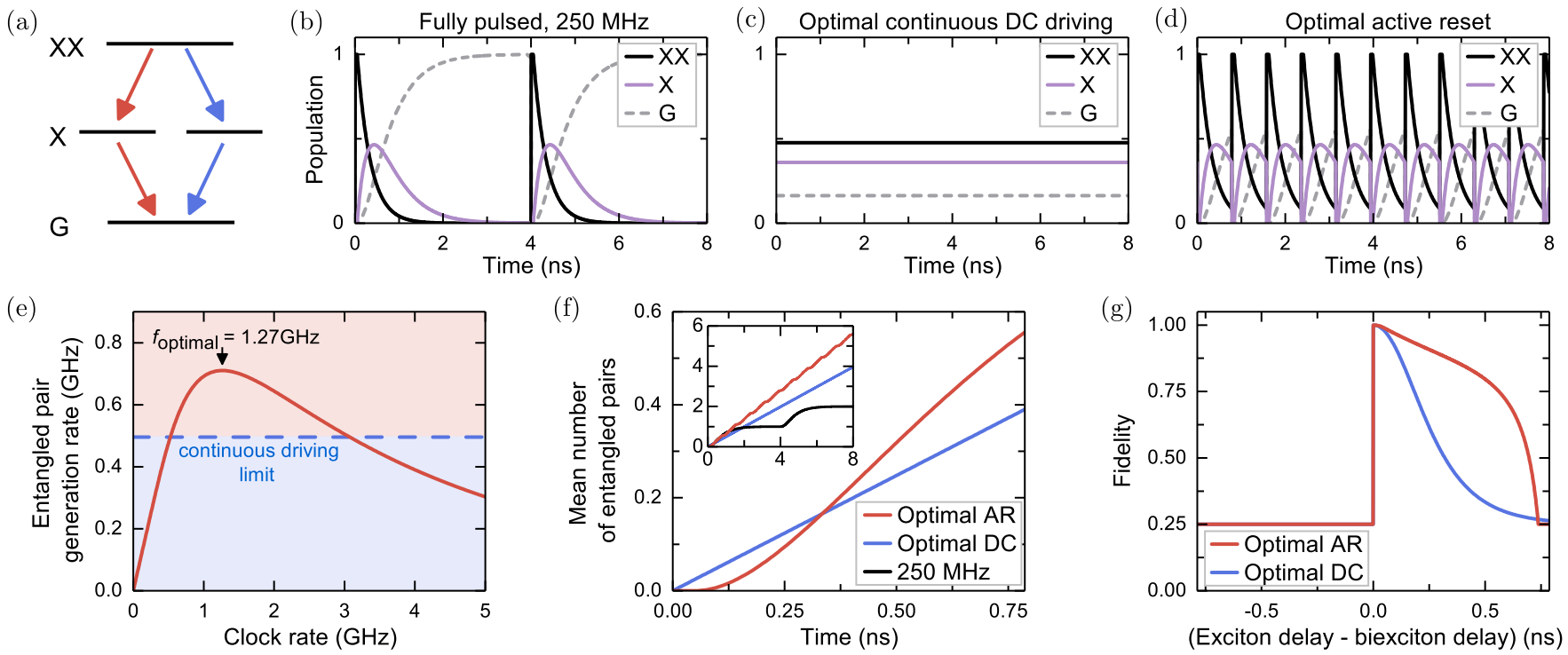

FIG. 1. Active reset (AR) of the radiative cascade. (a) Level diagram of the biexciton-exciton radiative cascade. Due to the superposition of the two separate decay paths (red and blue), the emitted photon pair forms a polarization-entangled state. [(b)-(d)] QD populations for different driving modes, calculated via a rate equation model. (b) Conventional full-cycle pulsed driving. The QD is reinitialized after the population has reached zero. (c) Conventional continuous DC driving. Populations are shown for the DC pump rate that maximizes the entangled pair emission rate. (d) Active reset regime. The quantum dot is reinitialized into the biexciton state before the QD population reaches zero. (e) Modeled time-averaged entangled photon pair generation rate as a function of driving clock rate. An optimum clock rate is given at $1.27 \mathrm{GHz}$. The light-blue-shaded region indicates pair generation rates accessible in continuous driving, while the light-pink-shaded region indicates entangled pair generation rates beyond the continuous driving limit. (f) Mean number of entangled photon pairs emitted over one $1.27 \mathrm{GHz}$ period for the driving modes of (b)-(d). The inset shows the same for a longer time scale. (g) Fidelity to a maximally entangled Bell state vs emission delay between exciton and biexciton, for optimal AR driving (red) and optimal DC driving (blue). AR driving maintains a high fidelity even for a maximized pair emission rate.

as the low-brightness periods in the tail of the emission are eliminated.

Driven in the AR regime at a fast $1.27-\mathrm{GHz}$ clock rate [Fig. 1(d)], the QD is perpetually kept in an optically active state with high $X X$ or $X$ populations, and low ground state population below 0.56 , eliminating dark periods and resulting in a high emission brightness. For entangled photon pairs generated from solid state sources, the entanglement fidelity will typically reduce for an increasing emission delay between the two photons, owing to the interaction of the QD with the solid-state environment [20]. By reinitialising the QD state early, we avoid the generation of weakly entangled pairs at longer emission delays.

A major consideration for AR driving is the optimum clock rate required to maximize the number of entangled photon pairs over time [Fig. 1(e)]. Kantner et al. [21] have shown that for the generation of single photons, an optimum clock rate exists, after which the emission efficiency decreases. For the case of entangled photon pair generation, at clock periods longer than the lifetimes of both, biexciton and exciton, the entangled-pair generation rate naturally scales approximately linearly with the repetition rate. At faster clock rates, the pair generation rate in turn reduces due to the nonzero lifetime of the excitonic states, as the delayed emission of the entangled photon pair is interrupted prematurely before the radiative cascade completes.

Perhaps surprisingly, the figure shows that there exists a range of frequencies between $520 \mathrm{MHz}$ and $3.07 \mathrm{GHz}$ for which the entangled pair generation rate exceeds that for continuous driving. As the absolute entangled pair flux per unit time is a product of the clock rate times the emission probability per clock cycle, the increase in the former can more than compensate for the reduction in the latter. The entangled pair generation rate reaches a maximum at a clock rate of $f_{\text {optimal }}=1.27 \mathrm{GHz}$ where it exceeds the optimum continuously driven pair generation rate by $43 \%$ (generating 0.56 entangled pairs per clock cycle). Even the highest-clocked experimental demonstrations operate at clock rates below $500 \mathrm{MHz}$, with none approaching the optimum $[15,16]$. In practice, the overall efficiency is affected by various external factors as well, such as device electrical bandwidth and collection efficiency.

Lastly, for AR driving the entanglement fidelity is limited only by additional $X X$ emission during the short (50 ps) initialization pulse [Fig. 1(g)] and produces an overall fidelity of $89.4 \%$ over one clock cycle. Meanwhile, the fidelity for DC is fundamentally constrained by competing uncorrelated emission from continuous excitation, limited to an overall fidelity of only $72.7 \%$ for the same time period. Altogether, this model demonstrates the feasibility of overcoming the limits on entanglement fidelity and entangled-pair brightness imposed by continuous DC or full-cycle pulsed driving.

\section{HIGH-FREQUENCY QUANTUM DOT LED}

Accessing the regime of gigahertz-clocked generation of single and entangled photons is experimentally challenging. 

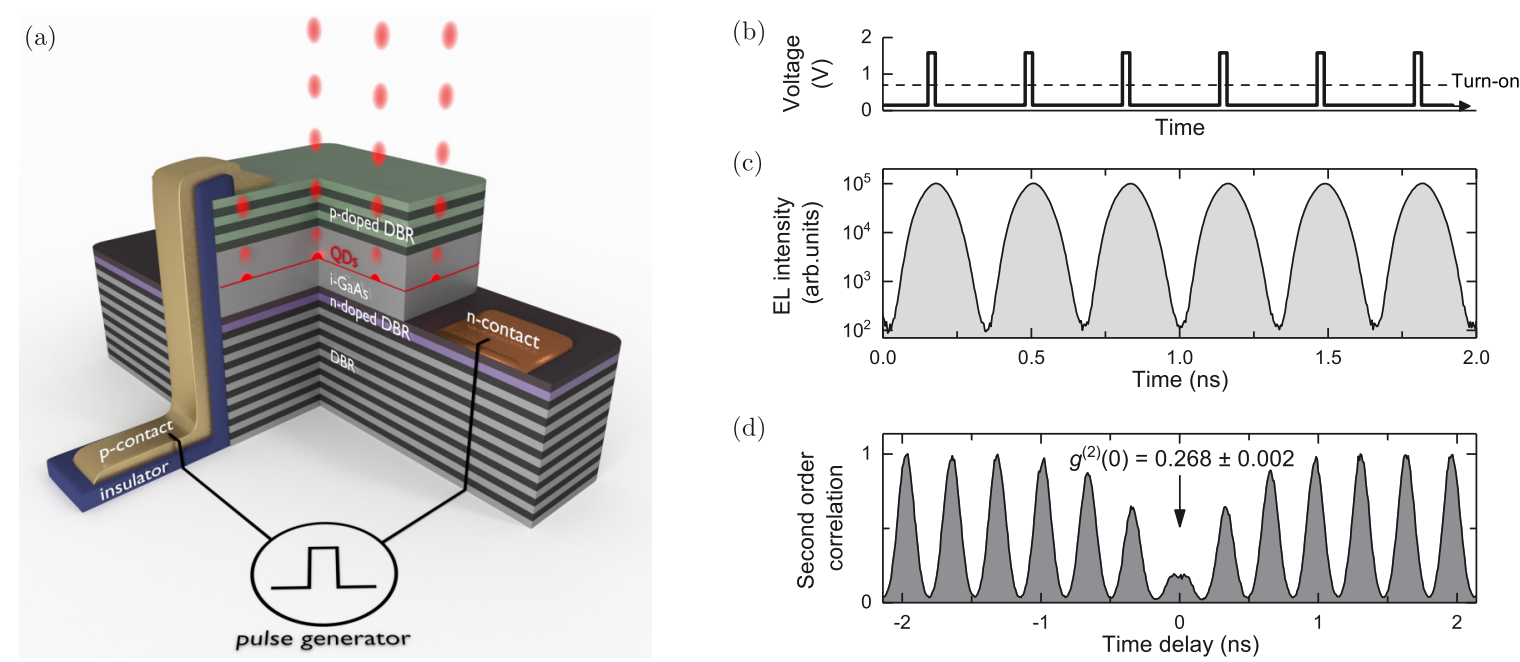

FIG. 2. Ultrafast electrical control of a QD LED. (a) Schematic illustration of the device cross section. Charge carriers are supplied to the QD by inducing an electrical current across the intrinsic region (i-GaAs). A small $10 \mu \mathrm{m} \times 10 \mu \mathrm{m}$ lateral size of the diode mesa and high-bandwidth optimized packaging (not shown) allow for GHz-clocked operation. (b) Nominal driving voltage pulses and (c) time-resolved neutral exciton electroluminescence intensity, driven at $3.05 \mathrm{GHz}$. (d) Second-order correlation $g^{(2)}(t)$ as a function of detection time delay. The emission yields a $g^{(2)}(0)$ value of $0.268 \pm 0.002$ for the integrated center peak.

Here we fabricate a high-bandwidth QD LED that responds to very short electrical pulses $<100$ ps [Fig. 2(a)]. InAs QDs were grown via molecular beam epitaxy (MBE) in the Stranski-Krastanov growth mode on a GaAs substrate and embedded in a $p-i-n$ diode structure, allowing for electrical charge carrier injection. Sets of 6(19) pairs of AlGaAs-GaAs DBR pairs above (below) the QD layer form a weak, planar $2 \lambda$ cavity, enhancing the collection efficiency of the emitted light. In order to facilitate $\mathrm{GHz}$ electrical control of the QD state, we etch the lateral dimensions of the diode mesa to an area of approximately $10 \mu \mathrm{m} \times 10 \mu \mathrm{m}$, nominally reducing the capacitance to $\sim 20 \mathrm{fF}$ in the case of a depleted diode. The $n$ - and $p$-type contacts are bonded to a custom designed printed circuit board (PCB) for electrical access. The $\mathrm{PCB}$ is single-sided and interfaces an array of microcoaxial connectors with impedance-matched ground-signal-ground transmission lines on FR4 glass epoxy for high frequency operation.

To verify the high bandwidth of the optimized device design, we investigate the optical signal when applying high frequency voltage pulses. The device was driven at $3.05 \mathrm{GHz}$ with driving pulses of amplitude $V_{\text {pulse }}=1.43 \mathrm{~V}$ and nominal duty cycle of $7.5 \%(\sim 25 \mathrm{ps})$, at a temperature of $6 \mathrm{~K}$. A DC bias voltage of $V_{\mathrm{DC}}=0.15 \mathrm{~V}$ is chosen to be far below the turn-on voltage $V_{T}=0.70 \mathrm{~V}$ of the diode [Fig. 2(b)]. Thus, charge carriers quickly tunnel out of the QD potential between driving pulses, quenching the optical emission [22,23] and revealing the electrical bandwidth of the device.

In a time-resolved measurement of the $X$ electroluminescence [Fig. 2(c)], we observe high contrast optical pulses with almost three orders of magnitude modulation of the emission intensity. Approximating the optical emission pulse with a Gaussian temporal shape yields a FWHM of $(100 \pm 2) \mathrm{ps}$, and fitting an exponential decay yields a lifetime of approximately 15 ps. This is in stark contrast to a 500-ps radiative lifetime, suggesting nonradiative decay processes are dominant, attributed to efficient tunneling of the charge carriers out of the QD enabled by fast electrical response.

The corresponding second-order autocorrelation $g^{(2)}(t)$, measured for the same driving conditions, is shown in Fig. 2(d). The value $g^{(2)}(0)=0.268 \pm 0.002$ of the zerodelay peak clearly demonstrates the single photon character of the emission. We attribute the nonzero component of the $g^{(2)}(0)$ value to re-excitation within the short driving pulse and background wetting layer emission (see Appendix B). The noticeable antibunching of the peaks adjacent to the zerodelay could imply the existence of long-lived charged or dark states [22]. To our knowledge, this is the fastest reported clock rate for the generation of single photons [23,24], entering the "super-high-frequency" radio band (comprising the $3-30 \mathrm{GHz}$ range [25]) for the first time.

\section{EXPERIMENTAL ACTIVE RESET}

After establishing ultrafast control of the QD single photon emission, we now turn our attention towards optimal generation of entangled photon pairs via AR driving, in a proof-of-principle experiment. The QD LED was electrically excited at a clock rate of $1.15 \mathrm{GHz}$ ( $T=868 \mathrm{ps}$ period) with initialization pulses of amplitude $V_{\text {pulse }}=0.6 \mathrm{~V}$ and nominal duty cycle $5 \%(\sim 43 \mathrm{ps})$, plus a DC bias of $V_{\mathrm{DC}}=0.6 \mathrm{~V}$ just below the turn-on voltage [Fig. 3(a)]. Further details in Appendix $C$ ). The resulting normalized biexciton-exciton second-order correlation function $g^{(2)}\left(t_{X X}, t_{X}\right)$ is shown in Fig. 3(b) as a function of $X X$ emission time $\left(t_{X X}\right)$ and $X$ emission time $\left(t_{X}\right)$ relative to the clock of the electrical driving pulse generator. Due to the cascaded emission of the $X$ photon after the $X X$ photon, the correlation events within a clock cycle (dashed black square) are naturally biased towards the upper triangle region where $t_{X}>t_{X X}$.

Figure 3(c) shows the fidelity to a maximally entangled evolving Bell state, extracted from co- and 
(a)

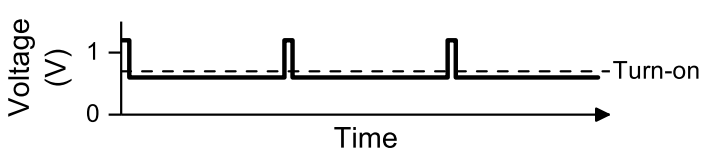

(b)

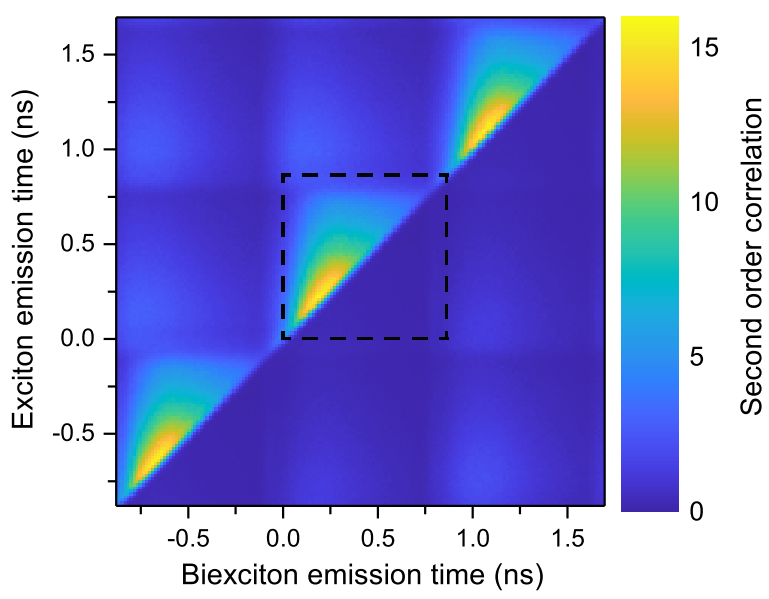

(c)

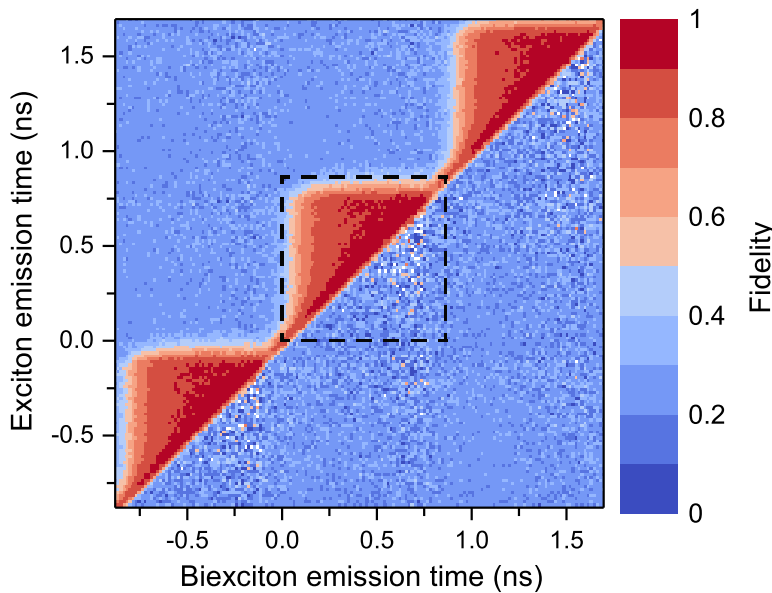

FIG. 3. Generation of entangled photon pairs at a $1.15 \mathrm{GHz}$ repetition rate. The dashed black square highlights photon pairs emitted within the same driving cycle. (a) Nominal driving voltage pulses. (b) Unpolarized biexciton-exciton second-order correlation $g^{(2)}\left(t_{X X}, t_{X}\right)$ as a function of biexciton emission time $t_{X X}$ and exciton emission time $t_{X}$. (c) Corresponding fidelity to a maximally entangled evolving Bell state $f\left(t_{X X}, t_{X}\right)$. The fidelity remains highly entangled within the driving cycle, and is sharply quenched towards the end of the cycle $t_{X} \rightarrow T$. The total fidelity within a cycle is $(79.5 \pm 1.1) \%$. The data are plotted in bins of $16 \mathrm{ps} \times 16 \mathrm{ps}$; bins with insufficient photon counts are colored in white.

cross-polarized correlations measured in the respective polarization bases [26]. The emission reaches a maximum fidelity of $(95.8 \pm 1.3) \%$ and remains above the classical limit of $50 \%$ for the majority of the cycle, indicating electrically driven photon pair generation at a record $1.15 \mathrm{GHz}$ clock rate. The overall entanglement fidelity, integrated over all photon pairs detected within the same driving cycle (including the "lower triangle" at $\left.t_{X}<t_{X X}\right)$, comes to $F=(79.5 \pm 1.1) \%$. For an entanglement-based QKD protocol, this value yields a quantum bit error rate of $(13.6 \pm 0.7) \%$ [27], well within the $27.6 \%$ limit required for secure quantum communication
[28]. Notably, no postselection is required in this driving mode-weakly entangled photon pairs at longer time delays are avoided naturally, as the entanglement is actively reset at the beginning of each cycle. Finally, at the edges of the driving cycle, the entanglement is abruptly reduced towards the classically uncorrelated value of $25 \%$. Crucially, the measured overall entanglement fidelity exceeds the overall fidelity of a comparison DC measurement $F_{\mathrm{DC}}=(71.2 \pm 1.0) \%$ for the same time window, taken under the same experimental conditions (Appendix C).

An essential factor for the performance of QKD systems are independent polarizations for photons emitted across subsequent clock cycles, in order to maintain a low quantum bit error rate [27]. Figure 4(a) shows the mean entanglement fidelity as a function of the number of clock cycles between $X X$ photon and $X$ photon detection. Importantly, only photon pairs emitted within the same clock cycle are entangled. Photon pairs emitted across different clock cycles in turn yield a mean fidelity close to the $25 \%$ mark of fully uncorrelated light. This implies that the QD state is reset efficiently at the beginning of each cycle, such that the entanglement does not carry over from one clock cycle to the next. Though reinitialization to the $X X$ state is dominant, other unentangled initial states may be formed due to the statistical nature of nonresonant excitation such as charged excitons and biexcitons.

For pairs detected within the same clock cycle, the measured fidelity as a function of time delay between the two photons [shown in Fig. 4(b)] resembles the concave shape predicted via the rate equation model in Fig. 1(g). For time delays approaching the end of the 868 ps repetition period, entanglement is quickly quenched. Remarkably, as predicted the measured fidelity in AR driving remains nonclassical for longer time delays than in DC driving. At the same time, the fidelity in DC driving remains significantly above $25 \%$ at the end of the repetition period, thus carrying over an undesirable polarization correlation into the next clock cycle.

Finally, we compare the measured entangled photon pair intensity in AR and DC, accumulated as a function of time delay relative to the clock signal. The form of the curves shown in Fig. 4(c) resemble those in Fig. 1(f), with constant pair emission rate for $\mathrm{DC}$ driving, and for $\mathrm{AR}$ the rate is highest during the central part of the clock period. The measurements show an entangled photon pair rate enhanced by $(21 \pm 3) \%$ for $1.15 \mathrm{GHz}$ AR driving compared to continuous driving in DC. This is of similar order to the predicted $43 \%$ seen in Fig. 1(f), with differences attributed to more complex dynamics in the experiment compared to the simple illustrative model (Appendix C). However, the implications of this result are clear; resetting the biexciton state before the system is likely in the ground state achieves a higher entangled photon emission rate than sustaining a continuously driven equilibrium state.

\section{DISCUSSION}

The super-high-frequency driven $(3.05 \mathrm{GHz})$ single photon LED and active reset driven $(1.15 \mathrm{GHz})$ entangled LED results presented above impact other quantum emitter technology, particularly those based on QDs. For electrically driven devices, a key achievement is the high bandwidth 

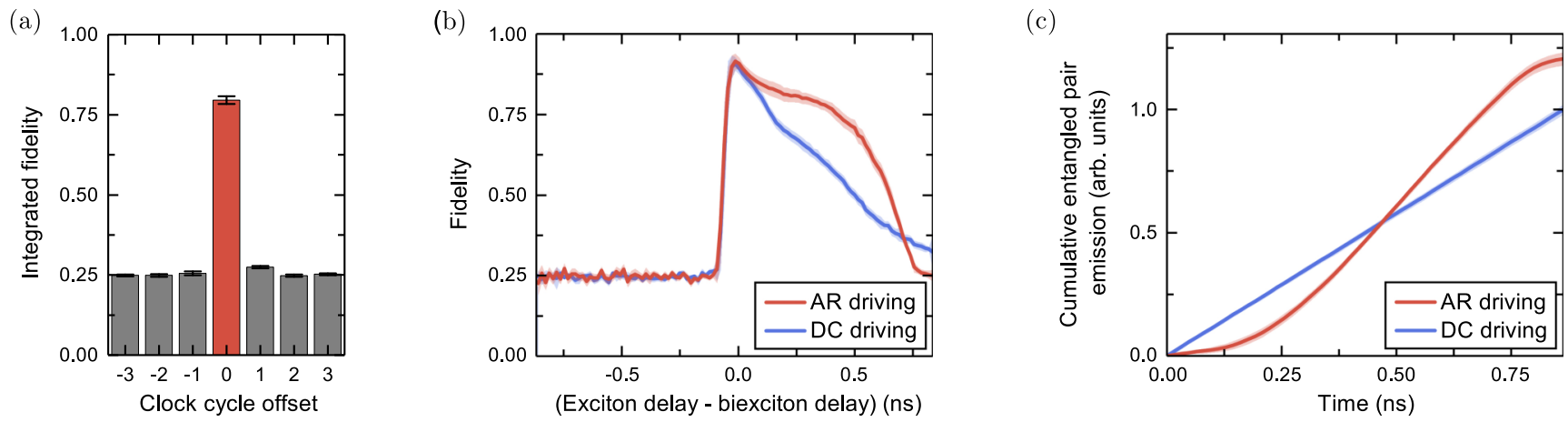

FIG. 4. Characteristics of active reset driving at $1.15 \mathrm{GHz}$. (a) Reliable reset of entanglement. Integrated entanglement fidelity as a function of clock cycles between biexciton emission and exciton emission. The integrated fidelity is nonclassical only for photon pairs emitted within the same clock cycle. (b) Comparison of the entanglement fidelity for AR and DC driving. Histogram of the measured entanglement fidelity as a function of the exciton photon detection delay after the biexciton photon, $t_{X-X X}=t_{X}-t_{X X}$. The fidelity in AR driving for coincidences emitted within the same cycle (red line) remains substantially higher than in DC driving (blue line) for the majority of the cycle. (c) Cumulative entangled pair emission. Plotted over one $1.15 \mathrm{GHz}$ cycle, normalized by the overall two-photon coincidences in the respective experiment. After one clock cycle, AR driving yields $(21 \pm 3) \%$ more entangled pairs. Error bars and shaded ribbons indicate the respective estimated standard error based on Poissonian detection statistics and estimated setup drifts in polarization.

entangled-LED mesa structure itself, which places no particular constraints on the optical device design or collection optics. The approach is therefore compatible with a wide variety of other techniques to enhance the source brightness, such as micropillars [3,29], broadband antennas [4], circular Bragg grating cavities $[7,8]$, or photonic crystals [30]. Notably, this driving scheme could benefit from Purcell-enhanced radiative decay rates some of these approaches provide, reducing the radiative lifetime and shifting the optimum AR clock rate to even higher frequencies.

Entangled LEDs operated in the active reset regime may benefit the overall performance of entanglement-based photonic applications. The gigahertz-clocked generation of entangled photon pairs combined with the enhanced entanglement fidelity and source brightness compared to continuous operation is of particular interest for entanglement-based QKD protocols, quantum relays, and future implementations of a quantum repeater.

\section{ACKNOWLEDGMENTS}

The authors thank Marco Lucamarini and Matthew Anderson for helpful discussions. This project has received partial funding from the European Union's Horizon 2020 research and innovation programme under the Marie SkłodowskaCurie grant Agreement No. 721394 and the Innovate UK project FQNet. JRAM thanks Alexander Tartakovskii for academic supervision.

\section{APPENDIX A: RATE EQUATION MODEL}

For the numerical modeling, the quantum dot is described as a four-level system, consisting of biexciton state $X X$, entangled neutral exciton state $X_{\text {ent }}$, nonentangled neutral exciton state $X_{\text {inc }}$ and ground state $G$. Here, $X_{\text {ent }}$ is defined as exciton state entangled to a previously radiatively emitted biexciton photon.
The rate equation model can be written as

$$
\begin{aligned}
\dot{\rho}_{X X} & =-\Gamma_{X X} \rho_{X X}+P_{X X}\left(\rho_{X, \text { inc }}+\rho_{X, \text { ent }}\right), \\
\dot{\rho}_{X, \text { inc }} & =-\left(P_{X X}+\Gamma_{X}\right) \rho_{X, \text { inc }}+P_{X} \rho_{G}, \\
\dot{\rho}_{X, \text { ent }} & =\Gamma_{X X} \rho_{X X}-\left(P_{X X}+\Gamma_{X}\right) \rho_{X, \text { ent }}, \\
\dot{\rho}_{G} & =\Gamma_{X}\left(\rho_{X, \text { inc }}+\rho_{X, \text { ent }}\right)-P_{X} \rho_{G},
\end{aligned}
$$

where $\rho$ is the respective population, $P_{X X / X}$ are the timedependent pump rates for the respective eigenstate, and $\Gamma_{X X / X}$ are the corresponding radiative decay rates. Inspection reveals the radiative decay rates limit the entangled pair generation rate, though as we show the limit for AR excitation strategies exceeds that for continuous driving. In the ideal active reset scheme, the diode is in the flat band condition in between driving pulses, thus tunneling of the charge carriers out of the quantum dot is assumed to be negligible. The time-dependent emission rate of polarization-entangled photon pairs corresponds to $\Gamma_{\text {pair }}=\Gamma_{X} \rho_{X \text {,ent }}$.

For Fig. 1 of the main text, the radiative decay rates are determined by the respective lifetimes at the diode turn-on voltage, as displayed in Figs. 6(a) and 6(b). The pumping rate for AR driving is approximated as infinite during the 50-ps driving pulse. Spin scattering from $X_{\text {ent }}$ to $X_{\text {inc }}$ is omitted in this model (cf. Ref. [20]) - a significant spin scattering rate would increase $f_{\text {optimal }}$ as well. To find the optimal DC driving conditions for Fig. 1(d) of the main text, the pump rates $P_{X X}$ and $P_{X}$ are set to be equal [31]. Subsequently $\Gamma_{\text {pair }}$ as a function of $P_{X X}$ is maximized. If $P_{X X}$ is increased beyond the DC optimum, re-excitation of the $X_{\text {ent }}$ state to the $X X$ state dominates, reducing $X_{\text {ent }}$ and thus entangled pair emission.

The computation of the entanglement fidelity in DC driving plotted in Fig. $1(\mathrm{~g})$ of the main text follows the rate equation method described in Ref. [20]. To capture the time-dependent dynamics in AR driving, the two-photon density matrix is computed as a function of $\left(t_{X}, t_{X X}\right)$ within one clock cycle and subsequently integrated along $t_{X X}=t_{X}$. In this model, the 

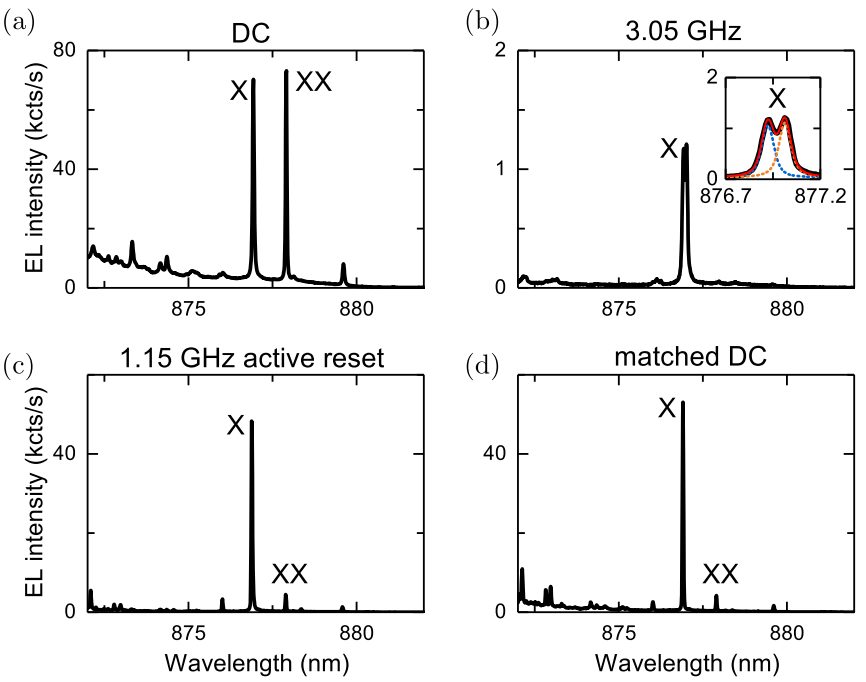

FIG. 5. EL spectra for different driving conditions. (a) Driven in DC near the $X$ saturation current. (b) Spectrum at the conditions for $3.05 \mathrm{GHz}$-clocked single photon emission. The inset shows a zoom-in on the exciton peak, revealing a split peak due to timedependent a Stark shift. The red line indicates a double Voigt curve fit, with the underlying Voigt curves marked by the dotted lines. (c) Spectrum at the chosen active reset conditions. (d) Spectrum at the reference DC conditions matched to the AR settings. Note that in AR driving the $X$ peak is slightly broadened compared to the DC settings, thus appearing lower despite a near-identical total intensity when integrated over the linewidth.

entanglement fidelity is limited only by competing emission from an incomplete radiative cascade.

\section{APPENDIX B: TIME-RESOLVED MEASUREMENT OF ELECTROLUMINESCENCE AND ENTANGLEMENT}

The sample was kept at $6 \mathrm{~K}$ in a vapor cryostat fitted with a confocal optical microscope setup. Combined with a low areal QD density $\left(<1 \mu \mathrm{m}^{-2}\right)$, this allows for coupling of emission from a selected single QD into single mode optical fiber. The $\mathrm{GHz}$-clocked electrical driving voltage pulses were supplied via a 3.35-GHz pulse generator. Electrical excitation of the QD eliminates the need for complex laser systems required for optical excitation.

The biexciton and exciton emission was centered at 876.8 and $877.8 \mathrm{~nm}$, respectively (Fig. 5) and spatially separated via a transmission grating. For all time-resolved measurements, the photons were detected via superconducting single photon detectors (SSPD) with an overall time jitter of 50-ps full width at half maximum (FWHM). Figures 6(a) and 6(b) show the time-resolved electroluminescence (EL) of the two transitions under excitation from a nominally 200-ps electrical driving pulse of $0.5-\mathrm{V}$ amplitude and a DC bias set to the turn-on voltage of $0.7 \mathrm{~V}$. Monoexponential fits reveal lifetimes of $\tau_{X X \text {,radiative }}=(300 \pm 1) \mathrm{ps}$ and $\tau_{X \text {,radiative }}=(499 \pm 1) \mathrm{ps}$, respectively.

For the $3.05-\mathrm{GHz}$ single photon generation in the main text (Fig. 2 of the main text), we reduced the DC bias to $0.15 \mathrm{~V}$, far below the turn-on voltage. This lead to a reduction in $X$ lifetime to approximately 15 ps.
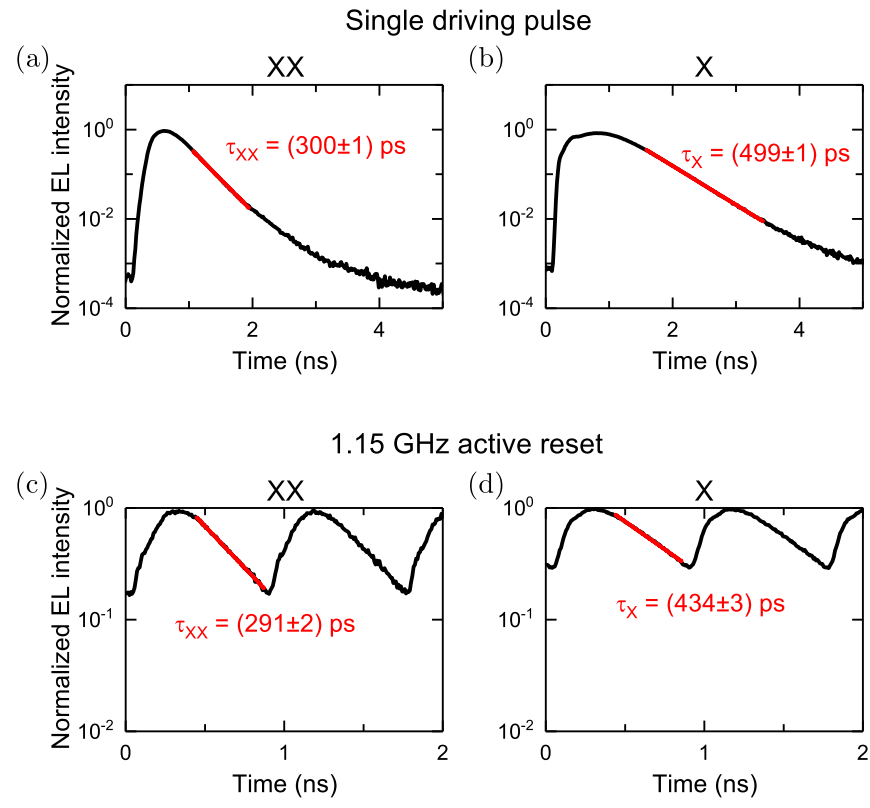

FIG. 6. EL intensity as a function of time. (a) Biexciton and (b) exciton emission line under excitation from a single $200 \mathrm{ps}$ electrical driving pulse. (c) Biexciton and (d) exciton emission line at the chosen active reset settings. In this case, the EL emission is reinitialized before the QD population reaches zero. Monoexponential fits used to extract the lifetimes are shown in red.

Assuming exponential tunneling and radiative recombination probabilities [23], we can estimate an upper limit for the fraction of excitons recombining radiatively via $\eta_{\text {radiative }}=$ $\tau_{\text {tunneling }} /\left(\tau_{\text {tunneling }}+\tau_{X, \text { radiative }}\right)=3 \%$. The remaining $97 \%$ recombine nonradiatively due to the high tunneling probability.

Under these driving conditions the $X$ emission peak splits into two components, emission during the driving pulses and emission after the driving pulses [Fig. 5(b), inset. Note that this splitting is much larger than the $10-\mu \mathrm{eV}$ fine structure splitting of the QD]. The latter is shifted to longer wavelengths as the reduced voltage between driving pulses induces a relative Stark shift [32]. By comparing the EL intensity of the two components, we can estimate an upper bound for the contribution of re-excitation during the driving pulse to the nonzero $g^{(2)}(0)$. According to the double Voigt curve fit to the spectrum, emission during (after) the driving pulse contributes $48 \%(52 \%)$ to the total $X$ emission.

In the limit of strong pumping to the $X$ state, such that re-excitation during the driving pulse is fast compared to the radiative lifetime, we can approximate a Poissonian contribution to the zero-delay peak of the $g^{(2)}$, written as the photon state [33]

$$
\left|\phi_{|\alpha|^{2}}\right\rangle=e^{-|\alpha|^{2} / 2} \sum_{n=0}^{\infty} \frac{\alpha^{n}}{\sqrt{n !}}|n\rangle,
$$

where $|\alpha|^{2}$ is the mean photon number. Any emission after the driving pulse is emitted in the single photon Fock state $|1\rangle$, as re-excitation is suppressed. With the total two-photon state in a clock period as a superposition of these two components, weighted by the respective relative contribution to the measured spectrum [and disregarding absolute scaling with 
respect to the vacuum state, as it does not affect the $g^{(2)}(0)$ value]

$$
|\psi\rangle=\left|\phi_{0.48}\right\rangle+0.52|1\rangle,
$$

we can calculate

$$
g^{(2)}(0)=\frac{\left\langle\psi\left|\hat{a}^{\dagger} \hat{a}^{\dagger} \hat{a} \hat{a}\right| \psi\right\rangle}{\left\langle\psi\left|\hat{a}^{\dagger} \hat{a}\right| \psi\right\rangle^{2}}=0.23
$$

for the zero-delay peak, where $\hat{a}^{\dagger}$ and $\hat{a}$ are the creation and annihilation operators, respectively. Comparing this to the measured $g^{(2)}(0)$ of $0.268 \pm 0.002$ as described in the main text, we conclude that re-excitation during the driving pulse is likely the dominating contribution to the nonzero $g^{(2)}$, with background emission constituting only a secondary factor. As is evident from the estimation here, the strong tunneling induced in this mode, reducing the intensity of the single photon emission, magnifies the relative contribution of re-excitation. Multiphoton emission due to re-excitation could potentially be lowered by further reducing the temporal duration of the initialization pulse. Note that finite carrier mobilities means that it takes on the order of $10 \mathrm{ps}$ to deplete the QD layer from charge carriers after the end of the electrical initialization pulse [21]. To what degree charge carriers would repopulate the QD during this time period then likely depends on the exact experimental settings.

In the second-order correlation $g^{(2)}$ in Fig. 2(c) of the main text, the coincidence counts are up to 4000 per time bin.

To evaluate the fidelity to a maximally entangled evolving Bell state, the collected photons were projected onto the desired polarization basis via electronically controlled polarization controllers (EPC) combined with fiber-coupled polarizing beam splitters (PBS) and recorded via SSPDs in 2 ps bins with respect to the pulse generator clock. The detection events were then downsampled to 16 ps bins and correlated in postprocessing.

To calculate the entanglement fidelity $F\left(t_{X X}, t_{X}\right)$ to an evolving Bell state, we use the relation [26]

$$
\begin{aligned}
F= & \frac{1}{4}\left[1+C_{\mathrm{HV}}+\left(C_{\mathrm{DA}}-C_{\mathrm{LR}}\right) \cos \left(S \frac{\tau_{X}-\tau_{X X}}{\hbar}\right)\right. \\
& \left.+\left(C_{\mathrm{E}_{\mathrm{LD}} E_{R A}}-C_{\mathrm{E}_{\mathrm{LA}} E_{R D}}\right) \sin \left(S \frac{\tau_{X}-\tau_{X X}}{\hbar}\right)\right],
\end{aligned}
$$

where $C_{\mu}$ are the degrees of correlation

$$
C_{\mu}=\frac{g_{X X, X}^{(2)}-g_{X X, \bar{X}}^{(2)}}{g_{X X, X}^{(2)}+g_{X X, \bar{X}}^{(2)}}
$$

using the copolarized $g_{X X, X}^{(2)}$ and cross-polarized $g_{X X, \bar{X}}^{(2)}$ biexciton-exciton second-order correlations, measured after projection of the photons onto the respective polarization basis $\mu . S$ is the fine structure splitting $(10 \mu \mathrm{eV}$ in this case) of the neutral exciton emission.

\section{APPENDIX C: ACTIVE RESET DRIVING CONDITIONS}

Figures 6(c) and 6(d) shows the time-resolved EL for the biexciton and exciton transitions under the chosen active reset driving conditions. The nominal DC bias in this mode was reduced to $0.6 \mathrm{~V}$ to ensure the diode remains below turn-on even at high frequency driving. A weak decrease of the $X$ lifetime [Fig. 6(d)] implies a low tunneling rate, while the $X X$ lifetime [Fig. 6(c)] remains largely unaffected. The emission intensity for both transitions remains nonzero throughout, analogous to the proposed situation in Fig. 1(b) of the main text. The clock rate of $1.15 \mathrm{GHz}$ chosen for experimental AR was reduced from the theoretical optimum of $1.27 \mathrm{GHz}$ to ease requirements on the device bandwidth and reduce distortion via electrical resonances. For this reduced clock rate, the theoretical model still predicts an entangled pair generation rate of more than $99 \%$ of the optimal value [cf. Fig. 1(e) of the main text]. To mitigate the contribution of background and competing QD emission in the employed sample, the $\mathrm{AR}$ and $\mathrm{DC}$ experiments were performed at reduced pumping intensity from the predicted optimum. The DC current was set to $27 \%$ of the $X$ saturation current, or approximately $17 \%$ of the predicted optimum DC pumping intensity. The driving voltage for the AR measurement was chosen such that the resulting integrated exciton and biexciton emission lines reach $100.4 \%$ and $115.5 \%$ intensity, respectively, compared to the DC setting [Figs. 5(c) and 5(d)]. The number of entangled photon pairs is estimated by approximating the QD emission as a mixed state consisting of maximally entangled, and uncorrelated photon pairs. In other words, only those pairs where both photons of a completed biexciton-exciton cascade are detected contribute to the number of entangled photons. The data were normalized by the total number of detected two-photon coincidences in the respective experimental run, such that the number of entangled pairs can be compared for the same emission intensity in both AR and DC. This compensates for the slightly reduced biexciton intensity in the matched DC driving mode as well as any drifts in setup efficiency between the two runs. The absolute detected entangled pair rate was $(156 \pm 3) \mathrm{Hz}$ for the DC equilibrium. For AR driving the entangled pair rate, after normalizing to the same number of total two-photon coincidences as in DC, was $(188 \pm 3) \mathrm{Hz}$. The detected entangled pair count rate scales with the square of the overall collection efficiency. Thus an optimized collection efficiency would bring the detection rates closer to the near-GHz internal entangled pair generation rates of the QD system itself.

When matching the DC exciton intensity to AR at the employed driving intensities, the theoretically predicted relative AR advantage is $11 \%$. Thus we expect to reach a superior entangled pair generation rate in AR, when compared to the continuously DC driven equilibrium rate at the matched driving intensity. We attribute the increased experimentally measured advantage of $(21 \pm 3) \%$ to higher charge noise in DC due to the continuous electrical current. Thus the presented proof-of-principle experiment is a reliable predictor for the expected performance advantage at optimum driving parameters for a QD sample suitable for high pumping rates.

For comparison, Fig. 7 shows the experimentally measured DC driven second-order correlation and entanglement fidelity, plotted in the same way as Fig. 3 of the main text. In the DC measurement, the driving voltage is invariant over time, thus the second-order correlation [Fig. 7(a)] and the entanglement fidelity [Fig. 7(b)] are both translationally symmetric along the diagonal. In the main text, we quote the fidelity of $(71.2 \pm 1.0) \%$ over one $1.15-\mathrm{GHz}$ period of this 

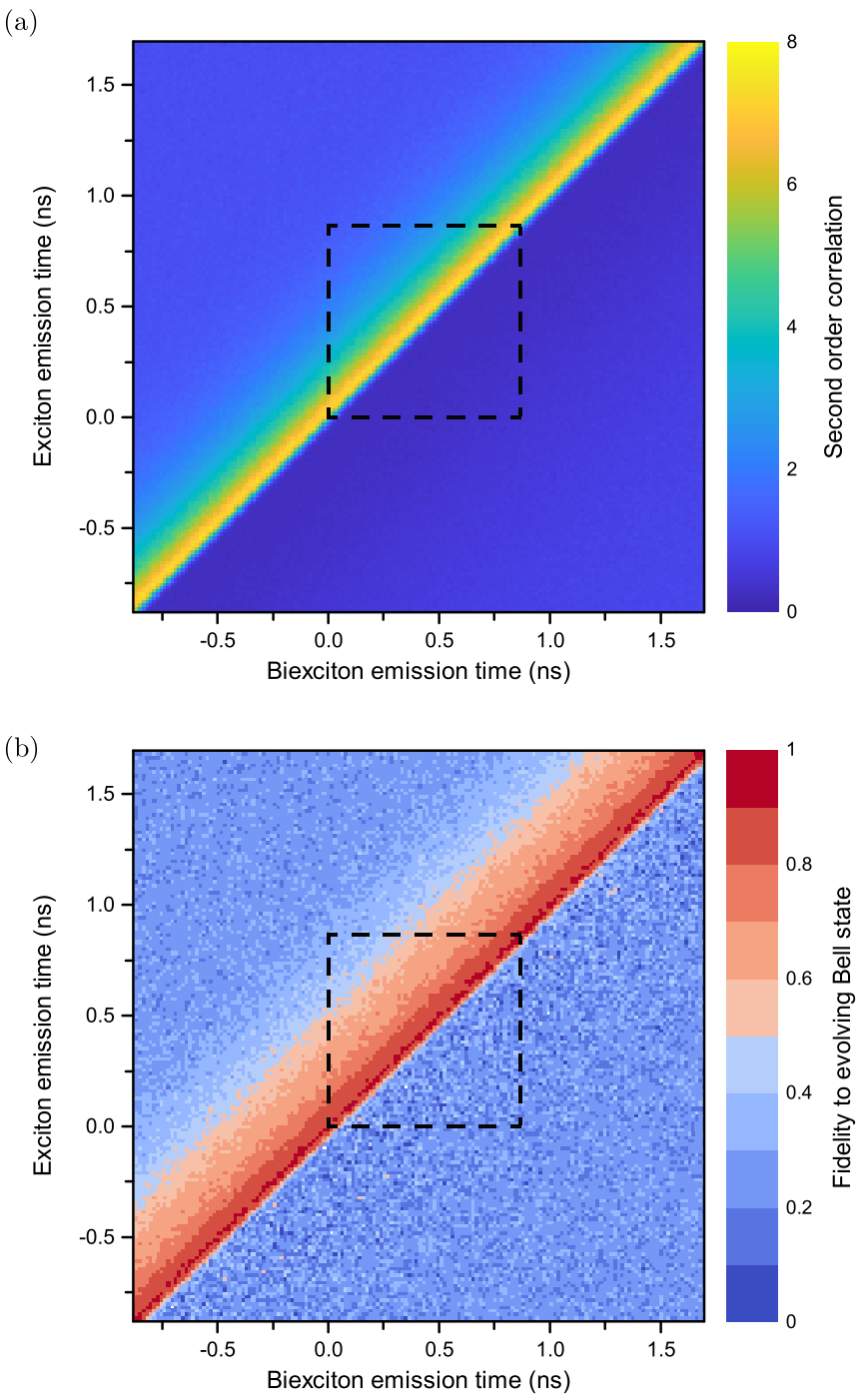

FIG. 7. Generation of entangled photon pairs using the DC driving parameters mentioned in the main text. For comparison with Fig. 3 of the main text. (a) Unpolarized biexciton-exciton secondorder correlation $g^{(2)}\left(t_{X X}, t_{X}\right)$ as a function of biexciton emission delay $t_{X X}$ and exciton emission delay $t_{X}$. (b) Corresponding fidelity to an evolving Bell state $f\left(t_{X X}, t_{X}\right)$. The total fidelity within a $1.15 \mathrm{GHz}$ period (dashed black square) is $(71.2 \pm 1.0) \%$.

measurement-we use this time frame solely as a comparison to the AR driving mode, as there is no inherent clock cycle in this measurement. The fidelity reaches a maximum value of $(92.5 \pm 1.9) \%$ in the $(16 \mathrm{ps} \times 16 \mathrm{ps})$ bin with the highest entanglement, similar to the maximum value of $(95.8 \pm 1.3) \%$ for AR driving mentioned in the main text. This is in agreement with the presented theoretical model, as neither driving mode limits the maximum entanglement fidelity at zero exciton-biexciton emission time delay [cf. Fig. 1(g) if the main text]. The dashed black square marks one period of the $1.15 \mathrm{GHz}$ clock cycle used in the respective AR driving measurement. In contrast to the AR data, both the unpolarized $g^{(2)}$ and the fidelity are no longer contained within the 868-ps periods.

\section{APPENDIX D: IMPACT OF SINGLE PHOTON PURITY ON ENTANGLED PHOTON GENERATION}

For optimal AR driving, we expect a significantly higher single photon purity for the invidual $X X$ and $X$ emission lines than in the $3.05 \mathrm{GHz}$ pulsed experiment. As tunneling is suppressed in AR driving, radiative recombination after the driving pulse is significantly increased. Consequently, the relative contribution of multiphoton contribution due to reexcitation is substantially reduced. For the optimum driving conditions modelled in Fig. 1 of the main text, re-excitation during the 43 ps nominal driving pulse adds an approximately Poissonian $X X$ contribution to the zero-delay $g^{(2)}$ peak with a mean photon number of $n=43$ ps $\Gamma_{X X}=0.14$ per driving pulse, compared to $0.91 X X$ photons emitted between driving pulses. Analogously to Eqs. (B2) and (B3), we theoretically estimate a contribution of $g_{X X-X X}^{(2)}(0)=0.018$ from re-excitation. For the $X$ emission, multiphoton emission is completely suppressed in the optimum case, as during the driving pulse the $X$ state is immediately pumped to the $X X$ state before recombination can occur. In practice, background contributions may further reduce the single photon purity of both emission lines, however, this would likely apply approximately equally to AR driving and DC driving.

For the practical comparison of the entangled photon pair emission in AR and DC driving in Fig. 4(c) of the main text, the effect of multiphoton $X X$ and $X$ contributions is inherently taken into account. Naturally, detected multiphoton contributions beyond completed radiative cascades will not be part of an entangled photon pair and accordingly reduce the measured entanglement fidelity towards the uncorrelated value. As mentioned in Appendix $\mathrm{C}$ above, we extract the fraction of entangled photon pairs from the experimental data via the fidelity in a given time bin, by approximating the QD emission as a mixed state, consisting of maximally entangled and uncorrelated photon pairs. This is then weighted by the $X X-X$ coincidence rate in the same time bin, normalized by the total number of detected two-photon coincidences. As a result, this comparison of entangled pair emission inherently corrects for nonentangled multiphoton contributions as well.

\section{APPENDIX E: COMPATIBILITY WITH OPTICAL BIEXCITON INITIALIZATION}

The presented AR scheme and the associated benefits raise ample opportunity for further investigation. Notably, the scheme could be combined with optical pumping of the biexciton state instead of electrical pumping. In particular two-photon excitation has been shown to enable high-fidelity generation of entangled photons from quantum dots [10]. In the AR scheme, the only partially populated ground state hinders a full population transfer to the biexciton state purely via Rabi flopping. However, compatible alternatives are given by two-photon excitation via adiabatic rapid passage [34,35] and phonon-assisted two-photon excitation [36-38], both of which are in principle capable of transferring partial ground and exciton populations to the biexciton state. 
[1] N. Gisin, G. Ribordy, W. Tittel, and H. Zbinden, Quantum cryptography, Rev. Mod. Phys. 74, 145 (2002).

[2] W. Dür, H.-J. Briegel, J. I. Cirac, and P. Zoller, Quantum repeaters based on entanglement purification, Phys. Rev. A 59, 169 (1999).

[3] X. Ding, Y. He, Z.-C. Duan, N. Gregersen, M.-C. Chen, S. Unsleber, S. Maier, C. Schneider, M. Kamp, S. Höfling, C.-Y. Lu, and J.-W. Pan, On-Demand Single Photons with High Extraction Efficiency and Near-Unity Indistinguishability from a Resonantly Driven Quantum Dot in a Micropillar, Phys. Rev. Lett. 116, 020401 (2016).

[4] Y. Chen, M. Zopf, R. Keil, F. Ding, and O. G. Schmidt, Highlyefficient extraction of entangled photons from quantum dots using a broadband optical antenna, Nat. Commun. 9, 2994 (2018).

[5] L. Hanschke, K. A. Fischer, S. Appel, D. Lukin, J. Wierzbowski, S. Sun, R. Trivedi, J. Vučković, J. J. Finley, and K. Müller, Quantum dot single-photon sources with ultra-low multi-photon probability, npj Quantum Inf. 4, 1026 (2018).

[6] D. Huber, M. Reindl, S. F. Covre da Silva, C. Schimpf, J. Martín-Sánchez, H. Huang, G. Piredda, J. Edlinger, A. Rastelli, and R. Trotta, Strain-Tunable GaAs Quantum Dot: A Nearly Dephasing-Free Source of Entangled Photon Pairs on Demand: An On-Demand Source of Nearly-Maximally Entangled Photon Pairs, Phys. Rev. Lett. 121, 033902 (2018).

[7] J. Liu, R. Su, Y. Wei, B. Yao, S. F. C. d. Silva, Y. Yu, J. Iles-Smith, K. Srinivasan, A. Rastelli, J. Li, and X. Wang, A solid-state source of strongly entangled photon pairs with high brightness and indistinguishability, Nat. Nanotechnol. 14, 586 (2019).

[8] H. Wang, H. Hu, T.-H. Chung, J. Qin, X. Yang, J.-P. Li, R.-Z. Liu, H.-S. Zhong, Y.-M. He, X. Ding, Y.-H. Deng, Q. Dai, Y.-H. Huo, S. Höfling, C.-Y. Lu, and J.-W. Pan, On-Demand Semiconductor Source of Entangled Photons Which Simultaneously Has High Fidelity, Efficiency, and Indistinguishability, Phys. Rev. Lett. 122, 113602 (2019).

[9] A. J. Shields, Semiconductor quantum light sources, Nat. Photonics 1, 215 (2007).

[10] D. Huber, M. Reindl, J. Aberl, A. Rastelli, and R. Trotta, Semiconductor quantum dots as an ideal source of polarizationentangled photon pairs on-demand: A review, J. Opt. 20, 073002 (2018).

[11] F. Olbrich, J. Höschele, M. Müller, J. Kettler, S. Luca Portalupi, M. Paul, M. Jetter, and P. Michler, Polarization-entangled photons from an InGaAs-based quantum dot emitting in the telecom C-band, Appl. Phys. Lett. 111, 133106 (2017).

[12] Z.-H. Xiang, J. Huwer, R. M. Stevenson, J. Skiba-Szymanska, M. B. Ward, I. Farrer, D. A. Ritchie, and A. J. Shields, Longterm transmission of entangled photons from a single quantum dot over deployed fiber, Sci. Rep. 9, 4111 (2019).

[13] Z. Yuan, A. Plews, R. Takahashi, K. Doi, W. Tam, A. W. Sharpe, A. R. Dixon, E. Lavelle, J. F. Dynes, A. Murakami, M. Kujiraoka, M. Lucamarini, Y. Tanizawa, H. Sato, and A. J. Shields, 10-Mb/s quantum key distribution, J. Lightwave Technol. 36, 3427 (2018).

[14] A. Boaron, G. Boso, D. Rusca, C. Vulliez, C. Autebert, M. Caloz, M. Perrenoud, G. Gras, F. Bussières, M.-J. Li, D. Nolan, A. Martin, and H. Zbinden, Secure Quantum Key Distribution over 421 km of Optical Fiber, Phys. Rev. Lett. 121, 190502 (2018).
[15] R. M. Stevenson, C. L. Salter, J. Nilsson, A. J. Bennett, M. B. Ward, I. Farrer, D. A. Ritchie, and A. J. Shields, Indistinguishable Entangled Photons Generated by a Light-Emitting Diode, Phys. Rev. Lett. 108, 040503 (2012).

[16] J. Zhang, J. S. Wildmann, F. Ding, R. Trotta, Y. Huo, E. Zallo, D. Huber, A. Rastelli, and O. G. Schmidt, High yield and ultrafast sources of electrically triggered entangled-photon pairs based on strain-tunable quantum dots, Nat. Commun. 6, 10067 (2015).

[17] For use of the cascade in single atoms see for example A Aspect, P. Grangier, and G. Roger, Experimental Tests of Realistic Local Theories via Bell's Theorem, Phys. Rev. Lett. 47, 460 (1981)

[18] O. Benson, C. Santori, M. Pelton, and Y. Yamamoto, Regulated and Entangled Photons from a Single Quantum Dot, Phys. Rev. Lett. 84, 2513 (2000).

[19] R. M. Stevenson, R. J. Young, P. Atkinson, K. Cooper, D. A. Ritchie, and A. J. Shields, A semiconductor source of triggered entangled photon pairs, Nature (London) 439, 179 (2006).

[20] R. M. Stevenson, A. J. Bennett, and A. J. Shields, Electrically operated entangled light sources based on quantum dots, in Quantum dots, edited by A. G. Tartakovskii (Cambridge University Press, Cambridge and New York, 2012), pp. 319-340.

[21] M. Kantner, M. Mittnenzweig, and T. Koprucki, Hybrid quantum-classical modeling of quantum dot devices, Phys. Rev. B 96, 205301 (2017).

[22] A. J. Bennett, D. C. Unitt, P. See, A. J. Shields, P. Atkinson, K Cooper, and D. A. Ritchie, Electrical control of the uncertainty in the time of single photon emission events, Phys. Rev. B 72, 033316 (2005).

[23] F. Hargart, C. A. Kessler, T. Schwarzbäck, E. Koroknay, S Weidenfeld, M. Jetter, and P. Michler, Electrically driven quantum dot single-photon source at $2 \mathrm{GHz}$ excitation repetition rate with ultra-low emission time jitter, Appl. Phys. Lett. 102, 011126 (2013).

[24] S. Buckley, K. Rivoire, and J. Vučković, Engineered quantum dot single-photon sources, Rep. Prog. Phys. 75, 126503 (2012).

[25] International Telecommunication Union, Nomenclature of the frequency and wavelengh bands used in telecommunications: Recommendation ITU-R V.431-8 (2015).

[26] M. B. Ward, M. C. Dean, R. M. Stevenson, A. J. Bennett, D. J. P. Ellis, K. Cooper, I. Farrer, C. A. Nicoll, D. A. Ritchie, and A. J. Shields, Coherent dynamics of a telecom-wavelength entangled photon source, Nat. Commun. 5, 3316 (2014).

[27] V. Scarani, H. Bechmann-Pasquinucci, N. J. Cerf, M. Dušek, N. Lütkenhaus, and M. Peev, The security of practical quantum key distribution, Rev. Mod. Phys. 81, 1301 (2009).

[28] H. F. Chau, Practical scheme to share a secret key through a quantum channel with a $27.6 \%$ bit error rate, Phys. Rev. A 66 , 060302(R) (2002).

[29] A. Dousse, J. Suffczyński, A. Beveratos, O. Krebs, A. Lemaître, I. Sagnes, J. Bloch, P. Voisin, and P. Senellart, Ultrabright source of entangled photon pairs, Nature (London) 466, 217 (2010).

[30] M. Arcari, I. Söllner, A. Javadi, S. Lindskov Hansen, S. Mahmoodian, J. Liu, H. Thyrrestrup, E. H. Lee, J. D. Song, S. Stobbe, and P. Lodahl, Near-Unity Coupling Efficiency of a Quantum Emitter to a Photonic Crystal Waveguide, Phys. Rev. Lett. 113, 093603 (2014). 
[31] T. Heindel, A. Thoma, M. von Helversen, M. Schmidt, A. Schlehahn, M. Gschrey, P. Schnauber, J.-H. Schulze, A. Strittmatter, J. Beyer, S. Rodt, A. Carmele, A. Knorr, and S. Reitzenstein, A bright triggered twin-photon source in the solid state, Nat. Commun. 8, 14870 (2017).

[32] M. B. Ward, T. Farrow, P. See, Z. L. Yuan, O. Z. Karimov, A. J. Bennett, A. J. Shields, P. Atkinson, K. Cooper, and D. A. Ritchie, Electrically driven telecommunication wavelength single-photon source, Appl. Phys. Lett. 90, 063512 (2007).

[33] M. O. Scully and M. S. Zubairy, Quantum Optics (Cambridge University Press, Cambridge, 1997).

[34] M. Glässl, A. M. Barth, K. Gawarecki, P. Machnikowski, M. D. Croitoru, S. Lüker, D. E. Reiter, T. Kuhn, and V. M. Axt, Biexciton state preparation in a quantum dot via adiabatic rapid passage: Comparison between two control protocols and impact of phonon-induced dephasing, Phys. Rev. B 87, 085303 (2013).
[35] T. Kaldewey, S. Lüker, A. V. Kuhlmann, S. R. Valentin, A. Ludwig, A. D. Wieck, D. E. Reiter, T. Kuhn, and R. J. Warburton, Coherent and robust high-fidelity generation of a biexciton in a quantum dot by rapid adiabatic passage, Phys. Rev. B 95, 161302(R) (2017).

[36] M. Glässl, A. M. Barth, and V. M. Axt, Proposed Robust and High-Fidelity Preparation of Excitons and Biexcitons in Semiconductor Quantum Dots Making Active Use of Phonons, Phys. Rev. Lett. 110, 147401 (2013).

[37] P.-L. Ardelt, L. Hanschke, K. A. Fischer, K. Müller, A Kleinkauf, M. Koller, A. Bechtold, T. Simmet, J. Wierzbowski, H. Riedl, G. Abstreiter, and J. J. Finley, Dissipative preparation of the exciton and biexciton in self-assembled quantum dots on picosecond time scales, Phys. Rev. B 90, 241404(R) (2014).

[38] S. Bounouar, M. Müller, A. M. Barth, M. Glässl, V. M. Axt, and P. Michler, Phonon-assisted robust and deterministic twophoton biexciton preparation in a quantum dot, Phys. Rev. B 91, 161302(R) (2015). 